\title{
Overview on the Phenotypic Drug Resistance in Mycobacterium Tuberculosis
}

\author{
Sultan Abda Neja* \\ Hawassa University, Faculty of Veterinary Medicine, Ethiopia
}

*Corresponding Author: Sultan Abda Neja, Hawassa University, Faculty of Veterinary Medicine, Ethiopia

\begin{abstract}
The modern lengthy treatments of tuberculosis (TB) patients with the most common regimen often have good clinical response within the first two weeks. But a population of phenotypically antibiotic resistant bacteria persists in a viable state. The pathophysiological conditions that potentially drive such phenotypic drug resistance and tolerance include the in vivo biofilm or complex physical barrier formation, persistence, stationary growth and state of dormancy. These events affects the duration of TB treatment and efficacy of anti-TB drugs. Additionally lack of good in vitro model to screen candidate compounds for such pathological condition also limited the anti-TB biofilm drug development. Moreover better understanding of TB pathogenesis remains to be a key approach for the development novel anti-TB drugs in foreseeable future. Hence, this manuscript gives an overview on the mechanisms by which persisters arise and the basis of their drug tolerance to underlay the phenotypic drug resistance in Mycobacterium tuberculosis.
\end{abstract}

\section{INTRODUCTION}

Antibiotic resistance can be broadly classified into genetic and phenotypic drug resistance. Genetic drug resistance is the most commonly studied and includes chromosomal mutations and acquisition of antibiotic resistance genes or transposons [1]. Phenotypic drug resistance includes resistance through altered drug access: influx, efflux, and deactivating enzymes, non-inherent change in bacterial growth, physiology and the formation of biofilms. Bacterial biofilms are complex communities of bacterial cells that develop on a solid surface [2]. They are made up of individually drug susceptible cells, but in response to various stresses, the biofilm start to forms and matures. This give rise to bacterial subpopulation with phenotypic heterogeneity that becomes refractory to drugs [3].

Human tuberculosis (TB) infections typically require treatment with multiple antibiotics for 6-9 months to avoid re-emergence of the disease [4]. In TB pathological lesion, subpopulation of bacteria often tolerates the conventional therapy and persists for long time. The inevitable reactivation of persistent drug-tolerant subpopulation of Mycobacterium tuberculosis (MTB) cells in tuberculous granuloma often develop to multiple drug-resistant (MDR) strain that exacerbate the challenges to control the spread of TB. Ideally the phenotypic drug resistances happening in TB lesion such as stationary growth phase or persistence and growth in dormant state are the typical characteristics of bacterial biofilms [5, 6]. Recent studies also showed MTB develops biofilm [1, 5, 7]; however detail mechanism in which TB-biofilm contribute to phenotypic drug resistance in MTB is not fully disclosed. Yet better understanding on TB-biofilm formation and associated drug tolerance has serious implication in future TB therapy.

\section{Mycobacterium Tuberculosis Phenotypic DRUg Resistance}

Phenotypic drug resistance and tolerance mechanism for MTB includes persistent and non-replicative states that allow the prolonged survival of the organism in the host environment. Briefly, in TB infection, the host immune response causes chronic inflammatory tissue reaction that leads to the formation of tissue granuloma. Within this granulomatous lesion of host micro-environment, there are varieties of triggers such as starvation, hypoxia, and reactive nitrogen or oxygen species. These stressors trigger a common signaling pathway, mediated by the dormancy survival (Dos) regulatory system, the sensor kinase DosS, and its cognate transcription factor DosR $[8,9,10]$. This is followed by the formation of layers of physical barrier (rich in mycolic acids, fats and cholesterol); low metabolic state and sub-population of tubercle bacilli starts to undergo deep dormancy. Such unique 
pathophysiological state along with other genetic factors like mmaA4 and Rv3852 that have been implicated in the pellicle form of MTB biofilm growth [11], may allow them to tolerate and survive thereby contributed to the long duration of treatment requirement to clear the infection.

\subsection{Persistence and Tolerance}

Phenotypic changes like growth arrest or stationary phase and metabolic quiescence often drive the cell's ability to survive antibiotic treatment, a phenomenon called drug tolerance. Cells surviving such condition are termed as persister cells. Recent review describes the mechanisms by which subpopulation of few bacteria persist and undergo drug tolerance include stochastic gene expression, swarming behavior, bacterial altruism, and phenotypic switching [2].

Asymmetric growth and division have recently been described in mycobacteria $[12,13,14]$ in which cells inheriting the older pole grow faster than cells inheriting the newer pole, and this creates dimorphism with each division. Faster growing cells are more susceptible to cell wall-acting antibiotics, while slower growing cells are more susceptible to transcription-targeting drugs [12]. Despite the potential clinical implications of persister cells [15], there is no consensus on the mechanisms by which persisters arise or the basis of their drug tolerance [16]. Further studies are needed in order to define their functional relevance in treatment failure.

\subsection{Biofilms in TB and its Implication in Future TB Therapy}

The presence of most obvious features of the bacterial biofilm and its similarities with the characteristics of MTB infections raised the question as to whether or not MTB forms biofilms in vivo, and whether these states can give rise to drug-tolerant persisters [17]. Driven by such question, recent preliminary evidence suggests that biofilms could perhaps be an in vivo lifestyle of MTB, contributing to their persistence against antibiotics [17-22]. In spite of these finding, such phenomenon is prominent in in vitro growth of M. smegmatis [23]. However as compared to in vitro biofilm, the contribution of host in the formation of district extracellular matrix of in vivo TB biofilm might make a difference in producing drug-tolerant microbial sub-population. As a result, the predictive value of in vitro biofilm model was limited and subsequently hampered the anti-TB biofilm drug development. Furthermore, the conditional phenotypic essentialities and the dynamic nature of drug interaction, metabolism in the host, pharmacokinetics within the bacilli as well as the unrevealed downstream events of killing concomitantly obscures the drug target.

For the future TB treatment, particularly to decrease the long durations of anti-TB treatment, it is important to reconsider the detail pathophysiology of TB biofilm formation. There are few recent findings which show the possibility to use compounds that are effective against drug-tolerant MTB and may further improve the biological activity as an adjunct to conventional antimicrobial therapy in vitro and in vivo [7]. Such additional compounds can be used to improve TB treatment by shortening the treatment interval and possibly increase drug concentrations required to effectively treat patients with both active and latent TB.

\section{CONCLUSION}

Phenotypic drug resistance is the phenomenon of transient changes of bacterial susceptibility to antibiotics. In MTB, there are different pathophysiological conditions that potentially drive the phenotypic drug resistence and tolerance. The in vivo biofilm or complex physical barrier formation, persistence, stationary growth and dormancy are among the events that affect the duration of TB treatment and efficacy of anti-TB drugs. Additionally lack of good in vitro model to screen candidate compounds for such pathological condition also limited the anti-TB biofilm drug development. Further studies are needed to unveil the mechanisms by which persisters arise and the basis of their drug tolerance as better understanding of TB pathophysiology remains to be a key approach for the development novel anti-TB drugs in foreseeable future.

\section{REFERENCES}

[1] Ojha A.K, Baughn A.D, Sambandan $\quad$ D, Hsu, T, Trivelli $\quad$ X, Guerardel $\quad$ Y. et al. (2008) Growth of Mycobacterium tuberculosis biofilms containing free mycolic acids and harbouring drug-tolerant bacteria. Mol Microbiol 69: 164-174.

[2] Kester J.C, Fortune S.M. (2013) Persisters and beyond: mechanisms of phenotypic drug resistance and drug tolerance in bacteria. Crit. Rev. Biochem. Mol. Biol. (Epub ahead of print). 
[3] Stewart P.S, and Costerton J.W. (2001). Antibiotic resistance of bacteria in biofilms. Lancet 358: 135-8.

[4] Hopewell P.C, Pai M., Maher D, Uplekar M, and Raviglione M.C. (2006) International standards for tuberculosis care. Lancet Infect. Dis. 6: 710-725.

[5] Corona F and Martinez J.L (2013) Phenotypic resistance to antibiotics. Antibiotics 2: 237-255.

[6] Zhang Y. (2007) Mechanisms of antibiotic resistance in the microbial world. Available at http://www. moleculartb.org/gb/pdf/transcriptions/11_YZhang.pdf. Baltimore, USA.

[7] Ackart D.F, Lindsey E.A, Podell B.K, Melander R.J, Basaraba R.J \& Melander C. (2014) Reversal of Mycobacterium tuberculosis phenotypic drug resistance by 2- aminoimidazole-based small molecules. Pathog. Dis. 70: 370-378

[8] Boon C. and Dick T. (2012) How Mycobacterium tuberculosis goes to sleep: the dormancy survival regulator DosR a decade later. Future Microbiol. 7: 513-518

[9] Kendall S.L, Movahedzadeh F, Rison SCG, et al. (2004). The Mycobacterium tuberculosis dosRS twocomponent system is induced by multiple stresses. Tuberculosis. 84: 247-55.

[10] Park H.D., Guinn KM, Harrell MI, et al. (2003). Rv3133c/dosR is a transcription factor that mediates the hypoxic response of Mycobacterium tuberculosis. Mol. Microbiol. 48: 833-43.

[11] Kerns P.W and Shirtliff, M.E. (2014) Development and Testing of a Five-Subunit Biofilm Vaccine for the Prevention of Pulmonary Tuberculosis. PhD thesis. University of Maryland School of Medicine, Baltimore, USA.

[12] Aldridge B.B, Fernandez-Suarez M, Heller D, et al. (2012). Asymmetry and aging of mycobacterial cells lead to variable growth and antibiotic susceptibility. Science 335:100-4.

[13] Joyce G, Williams K.J, Robb M, Noens E, Tizzano B, Shahrezaei V, and Robertson B. (2012) Cell division site placement and asymmetric growth in mycobacteria. PLoS ONE 7: e44582.

[14] Singh B, Nitharwal R.G, Ramesh M, et al. (2013). Asymmetric growth and division in Mycobacterium spp.: compensatory mechanisms for non-medial septa. Mol. Microbiol. 88: 64-76.

[15]Fauvart M, De Groote V.N and Michiels J. (2011) Role of persister cells in chronic infections: clinical relevance and perspectives on antipersister therapies. J. Med. Microbiol. 60: 699-709.

[16] Balaban NQ, Gerdes K, Lewis K, McKinney JD, et al. (2013). Perspectives. Nat. Rev. Microbiol. 11: 587-91.

[17] Islam M. S, Richards J. P, and Ojha A. K (2012) Targeting drug tolerance in mycobacteria: a perspective from mycobacterial biofilms. Expert Rev. Anti. Infect. Ther. 10(9): 1055-1066.

[18] Lenaerts AJ, Hoff D, Aly S, et al. (2007) Location of persisting mycobacteria in a Guinea pig model of tube rculosis revealed by r207910. Pathological evidence for the presence of drug-tolerant persisters of Mycobacterium tuberculosis in multicellular microcolonies. Antimicrob. Agents Chemother. 51(9): 3338-3345.

[19] Alteri CJ, Xicohténcatl-Cortes J, Hess S, Caballero-Olín G, Girón JA, Friedman RL. (2007) Mycobacterium tuberculosis produces pili during human infection. Proc Natl Acad Sci USA. 104(12): 5145-5150.

[20] Gill WP, Harik NS, Whiddon MR, Liao RP, Mittler JE, Sherman DR. (2009) A replication clock for Mycobacterium tuberculosis. Nat. Med. 15(2):211-214.

[21] Barry CE, 3rd, Boshoff HI, Dartois V, et al. (2009) The spectrum of latent tuberculosis: rethinking the biology and intervention strategies. Nat. Rev. Microbiol. 7(12): 845-855.

[22] Lin PL, Flynn JL. (2010) Understanding latent tuberculosis: a moving target. J Immunol. 185 (1):15-22.

[23] Teng R and Dick T (2003) Isoniazid resistance of exponentially growing Mycobacterium smegmatis biofilm culture. FEMS. Microbiol. Lett. 227(2): 171-4.

Citation: Sultan Abda Neja. "Overview on the Phenotypic Drug Resistance in Mycobacterium Tuberculosis ". International Journal of Research Studies in Microbiology and Biotechnology (IJRSMB), vol. 6, no. 2, pp. 3-5, 2020. Available: DOI: http://dx.doi.org/ 10.20431/2454-9428.0602002

Copyright: (C) 2020 Authors. This is an open-access article distributed under the terms of the Creative Commons Attribution License, which permits unrestricted use, distribution, and reproduction in any medium, provided the original author and source are credited. 